\title{
Factores de riesgo cardiovascular en Estados Unidos y México: comparación de los estudios HABLE y ENASEM
}

\author{
Raúl Vintimilla, *Miguel Reyes, Leigh Johnson, James Hall y Sid O’Bryant \\ Universidad del Norte de Texas, Centro de Ciencias de la Salud, Instituto de Investigación Traslacional, Texas, Estados Unidos
}

\section{Resumen}

Introducción: En Estados Unidos se dispone de información acerca de la población mexicoamericana por el Estudio de Salud y Envejecimiento del Cerebro en Latinos Mayores (HABLE); en México se dispone de los resultados del Estudio Nacional de Salud y Envejecimiento en México (ENASEM). Objetivo: Comparar la prevalencia de factores de riesgo cardiovascular entre hombres y mujeres de HABLE y ENASEM. Método: Se analizó transversalmente la prevalencia de hipertensión, diabetes, hipercolesterolemia y obesidad abdominal en 559 participantes de HABLE y se comparó con datos de 13663 participantes del ENASEM. La comparación se realizó mediante $t$ de Student y chi cuadrada, según el tipo de variable. Resultados: El análisis demostró que la prevalencia de hipertensión (50\%, IC $95 \%$ = 41.8-51.8), diabetes (35.5\%, IC $95 \%=27.6-43.8)$ y obesidad abdominal (59.3\%, IC $95 \%=50.5-68.1)$ fueron significativamente mayores en hombres del HABLE, mientras que las mujeres presentaron una prevalencia más elevada de diabetes (36.8 \%, IC 95 \% = 32.2-41.5) y obesidad abdominal (89.6 \%, IC $95 \%=86.6-92.5)$. La hipercolesterolemia tuvo una prevalencia más elevada en mujeres del ENASEM (53.3 \%, IC $95 \%$ = 50.3-56.2). Conclusión: La prevalencia de factores de riesgo cardiovascular fue mayor en mexicoamericanos participantes del HABLE, que en mexicanos participantes del ENASEM.

PALABRAS CLAVE: Enfermedad cardiovascular. Factores de riesgo. México. Mexicoamericanos.

\section{Cardiovascular risk factors in Mexico and the United States: a comparative cross-sectional study between the HABLE and MHAS participants}

\begin{abstract}
Introduction: In the United States, information on the Mexican-American population is available through the Health and Aging Brain among Latino Elders (HABLE) study; in Mexico, the results of the Mexican Health and Aging Study (MHAS) are available. Objective: To compare the prevalence of cardiovascular risk factors between men and women of the HABLE and MHAS studies. Method: The prevalence of hypertension, diabetes, hypercholesterolemia and abdominal obesity was transversely analyzed in 559 HABLE participants and compared with data from 13,663 MHAS participants. The comparison was made using Student's t-test and the chi-square test, according to the type of variable. Results: The analysis showed that the prevalence of hypertension (50\%, $95 \% \mathrm{Cl}=41.8-51.8)$, diabetes (35.5 \%, $95 \% \mathrm{Cl}=27.6-43.8$ ) and abdominal obesity (59.3 \%, $95 \% \mathrm{Cl}=50.5-68.1)$ were significantly higher in HABLE males, whereas females had a higher prevalence of diabetes (36.8 $\%, 95 \% \mathrm{Cl}=32.2-41.5)$ and abdominal obesity (89.6 \%, $95 \% \mathrm{Cl}=86.6-92.5)$. Hypercholesterolemia had a higher prevalence in MHAS females (53.3\%, 95\% Cl =50.3-56.2). Conclusion: The prevalence of cardiovascular risk factors was higher in Mexican American HABLE participants, than in Mexican MHAS participants.
\end{abstract}

KEY WORDS: Cardiovascular disease. Risk factors. Mexico. Mexican-Americans.

Correspondencia:

*Raúl Vintimilla

E-mail: raul.vintimilla@unthsc.edu CC BY-NC-ND (http://creativecommons.org/licenses/by-nc-nd/4.0/).
Fecha de recepción: 19-06-2019

Fecha de aceptación: 01-07-2019

DOI: 10.24875/GMM.19005350
Gac Med Mex. 2020;156:17-21

Disponible en PubMed

www.gacetamedicademexico.com 


\section{Introducción}

En Estados Unidos, las principales causas de muerte son las enfermedades cardiacas, el cáncer y las lesiones no intencionales. ${ }^{1}$ En mexicoamericanos, que constituyen $63.4 \%$ de latinos en ese país, ${ }^{2}$ el cáncer se ubica en el primer lugar, seguido de las enfermedades cardiacas y lesiones no intencionales. ${ }^{3}$ Datos del Instituto Nacional de Estadística y Geografía de México (INEGI) ubican a las enfermedades del corazón, la diabetes y el cáncer en los tres primeros lugares como causas de muerte en México. ${ }^{4}$ Desde hace varios años, la Organización Mundial de la Salud ha determinado que aproximadamente $31 \%$ de todas las muertes registradas se debe a enfermedades cardiovasculares, constituyéndose en la principal causa de muerte en todo el mundo. ${ }^{5}$

Debido al incremento de la esperanza de vida al nacer, un mayor porcentaje de personas envejecen y desarrollan enfermedades cardiovasculares, ${ }^{6}$ pero existen varios factores de riesgo como la hipertensión arterial sistémica, diabetes mellitus, dislipidemias y obesidad que pueden ser medidos, modificados o controlados. ${ }^{7}$ Estudios realizados en mexicoamericanos (sujetos nacidos en México que viven en Estados Unidos y sujetos de ancestro mexicano nacidos en Estados Unidos), como el San Antonio Heart Study o el Hispanic Community Health Study/Study of Latinos, han demostrado que la prevalencia de factores de riesgo cardiovascular (FRCV) es mayor en mexicoamericanos que en blancos no hispanos. ${ }^{8,9}$

En México, la Encuesta Nacional de Salud y Nutrición 2012 reportó una elevada prevalencia de FRCV en la población general, ${ }^{10}$ que concuerda con los resultados del estudio CARMELA ${ }^{11}$ y del realizado por Orozco González et al., en una muestra de trabajadores de salud en dos hospitales del Instituto Mexicano de Seguridad Social..$^{12}$

Varios investigadores han sugerido que los años de residencia en Estados Unidos, el lenguaje utilizado en el hogar y otros cambios en el comportamiento impactan de forma negativa en el estilo de vida de los mexicoamericanos, y coloca a esta población en riesgo mayor de desarrollar FRCV..$^{13,14}$ Sin embargo, debido a limitaciones metodológicas y a la inconsistencia en los métodos utilizados para cuantificar estas variables, los datos son contradictorios.

En el presente análisis comparamos la prevalencia de los FRCV en una muestra de mexicoamericanos que residen en Estados Unidos, que forma parte del estudio
Salud y Envejecimiento del Cerebro en Latinos Mayores (HABLE, Health \& Aging Brain among Latino Elders), con una muestra del Estudio Nacional de Salud y Envejecimiento en México (ENASEM). La hipótesis fue que los mexicoamericanos tienen mayor prevalencia de factores de riesgo cardiovascular que su contraparte mexicana. Entender las similitudes y diferencias en la distribución de estos factores de riesgo a ambos lados de la frontera es importante para desarrollar e implementar medidas efectivas de prevención.

\section{Método}

Estudio epidemiológico, descriptivo, transversal de dos poblaciones tomadas de dos estudios longitudinales: HABLE en Estados Unidos y ENASEM en México. HABLE analiza la relación de diferentes factores biológicos y enfermedades como diabetes, hipertensión, depresión, etcétera, con cambios en la memoria, conocimiento y envejecimiento en mexicoamericanos y blancos no hispanos. HABLE se lleva a cabo desde 2012 en el Centro de Ciencias de la Salud de la Universidad del Norte de Texas, en Fort Worth, Texas; recluta sujetos mayores de 50 años directamente de la comunidad, mediante reclutadores comunitarios, presentaciones, boletines de prensa, anuncios e información de boca en boca. Los participantes se someten a una entrevista detallada sobre información demográfica y salud, pruebas de evaluación neurosicológica, exámenes clínicos de sangre en ayunas, medidas antropométricas y a una evaluación médica.

Por su parte, el ENASEM es un estudio longitudinal nacional en México que se realiza desde el año 2001. La entrevista y recolección de datos se efectúa en personas mayores de 50 años, de zonas urbanas y rurales de los 32 estados del país. Este estudio es una colaboración entre la Universidad de Texas Medical Branch en Galveston, Texas, el Instituto Nacional de Estadística y Geografía y el Instituto Nacional de Salud Pública de México. El ENASEM estudia las enfermedades y discapacidad asociadas con el envejecimiento, evalúa el efecto del comportamiento individual, la migración, estatus socioeconómico y las características en la salud de la comunidad. $^{15}$ El cuestionario del ENASEM incluye información sobre datos demográficos, salud general, condiciones crónicas, estatus socioeconómico, migración, estructura familiar y condición de vivienda. En una submuestra de participantes se realizaron medidas antropomórficas, exámenes de sangre y pruebas neuropsicológicas. ${ }^{16}$ 
Tabla 1. Características demográficas según sexo

\begin{tabular}{|c|c|c|c|c|c|c|c|c|}
\hline & \multicolumn{4}{|c|}{ Hombres } & \multicolumn{4}{|c|}{ Mujeres } \\
\hline & \multicolumn{2}{|c|}{ HABLE $(n=138)$} & \multicolumn{2}{|c|}{ ENASEM $(n=5786)$} & \multicolumn{2}{|c|}{ HABLE $(n=421)$} & \multicolumn{2}{|c|}{ ENASEM $(n=7877$} \\
\hline & \multicolumn{2}{|c|}{ Media $\pm \mathrm{DE}$} & \multicolumn{2}{|c|}{ Media \pm DE } & \multicolumn{2}{|c|}{ Media \pm DE } & \multicolumn{2}{|c|}{ Media \pm DE } \\
\hline Edad (años) & \multicolumn{2}{|c|}{$63.9 \pm 8.3$} & \multicolumn{2}{|c|}{$68.2 \pm 15.5$} & \multicolumn{2}{|c|}{$60.4 \pm 8.2$} & \multicolumn{2}{|c|}{$67.0 \pm 17.8$} \\
\hline Educación (años) & \multicolumn{2}{|c|}{$7.7 \pm 4.8$} & \multicolumn{2}{|c|}{$6.1 \pm 5.8$} & \multicolumn{2}{|c|}{$7.7 \pm 4.2$} & \multicolumn{2}{|c|}{$5.2 \pm 5.1$} \\
\hline Estado civil casado & $\mathrm{n}$ & $\%$ & $\mathrm{n}$ & $\%$ & $\mathrm{n}$ & $\%$ & $\mathrm{n}$ & $\%$ \\
\hline Sí & 103 & 74.6 & 4580 & 87.8 & 202 & 48.2 & 4323 & 72.6 \\
\hline No & 33 & 25.4 & 636 & 12.2 & 217 & 51.8 & 1630 & 27.4 \\
\hline
\end{tabular}

HABLE $=$ Health \& Aging Brain among Latino Elders, ENASEM = Estudio Nacional de Salud y Envejecimiento en México.

Ambas investigaciones se apegaron a las condiciones éticas pertinentes para la investigación en seres humanos y fueron aprobadas por el comité de ética correspondiente. Todos los participantes firmaron un formato de consentimiento informado.

Entre mayo de 2012 y junio de 2015, 771 participantes fueron admitidos en el estudio HABLE, de los cuales, 559 eran de origen mexicano (138 hombres y 421 mujeres), residentes en el área de Dallas-Fort Worth, Texas; sus datos se utilizaron para el análisis. El rango de edad fue 50 a 85 años. Para el análisis de la población mexicana se accedió a la base de datos gratuita de la tercera ronda de ENASEM de 2012. ${ }^{17}$ Durante esta ronda se realizaron 18465 entrevistas. El análisis final sobre diabetes e hipertensión se realizó en 13663 participantes (5786 hombres y 7877 mujeres) y el análisis de colesterol total y obesidad abdominal en una submuestra de 1882 participantes (1112 mujeres). El rango de edad fue de 50 a 110 años.

El diagnóstico de hipertensión arterial y diabetes mellitus se adjudicó con base en el autorreporte de diagnóstico médico previo o uso de medicamentos para estas dos patologías. Conforme estándares establecidos, se consideraron niveles altos de colesterol a cifras $>200 \mathrm{mg} / \mathrm{dL}$ y obesidad abdominal a una circunferencia de la cintura $>40$ pulgadas en los hombre $y>35$ pulgadas en las mujeres.

\section{Análisis estadístico}

Se realizó un análisis descriptivo de todas las variables del estudio. Las características demográficas se presentan como media \pm desviación estándar para las variables continuas y como frecuencia y porcentaje para las variables categóricas. Se estableció la prevalencia de FRCV. La prevalencia se reporta en porcentajes con un intervalo de confianza de $95 \%$. La comparación entre grupos se realizó mediante $t$ de Student para variables continuas y $\chi^{2}$ para variables categóricas. El análisis se dividió por sexos. Una $p \leq 0.05$ se consideró significativa. Para el análisis se utilizó el programa estadístico SPSS para Windows, versión 23 (SPSSW Inc., Chicago, IL).

\section{Resultados}

Los hombres y mujeres del ENASEM tuvieron una edad media más elevada que la de los participantes del estudio HABLE, 68.2 y 67.0, respectivamente. (Tabla 1). Los participantes de HABLE tuvieron un nivel más alto de educación con una media de 7.7 años, tanto en hombres como en mujeres. Ochenta y ocho y $75 \%$ de los hombres de ENASEM y de HABLE reportaron estar casados; en las mujeres la diferencia fue mayor: $73 \%$ versus $48 \%$ en ENASEM y HABLE, respectivamente. Cabe destacar que todas las diferencias entre las muestras fueron estadísticamente significativas ( $p \leq 0.05)$.

La prevalencia de FRCV en hombres se aprecia en la Tabla 2. La prevalencia de hipertensión fue de $50 \%$ (IC $95 \%=41.8-51.8$ ), diabetes de $35.5 \%$ (IC $95 \%=$ 27.6-43.8) y obesidad abdominal de $59.3 \%$ (IC $95 \%$ = 50.5-68.1); el análisis demostró que las prevalencias en hombres de HABLE fueron significativamente mayores que en los de ENASEM. En hipercolesterolemia, la diferencia entre hombres de los dos estudios no fue estadísticamente significativa $(p=0.2)$.

La prevalencia de diabetes y obesidad abdominal también fue significativamente más elevada en las mujeres de HABLE, $36.8 \%$ (IC $95 \%=32.2-41.5$ ) y $89.6 \%$ (IC $95 \%=86.6-92.5$ ), respectivamente, comparadas con su contraparte de ENASEM (Tabla 3). En cuanto a la hipercolesterolemia, la prevalencia fue significativamente más elevada en las mujeres de ENASEM, $53.3 \%$ (IC $95 \%=50.3-56.2$ ). La diferencia 
Tabla 2. Prevalencia de factores de riesgo cardiovascular por sexo masculino

\begin{tabular}{|c|c|c|c|c|c|c|c|}
\hline & \multicolumn{3}{|c|}{ HABLE } & \multicolumn{3}{|c|}{ ENASEM } & \multirow[t]{2}{*}{$\mathbf{p}^{*}$} \\
\hline & $n$ & $\%$ & IC $95 \%$ & n & $\%$ & IC $95 \%$ & \\
\hline Hipertensión & 138 & 50.0 & $41.8-51.8$ & 5786 & 41.2 & $39.9-42.4$ & 0.03 \\
\hline Diabetes & 138 & 35.5 & $27.6-43.8$ & 5786 & 22.4 & $21.2-23.4$ & 0.0003 \\
\hline Hipercolesterolemia & 138 & 34.8 & $26.7-42.8$ & 770 & 40.6 & $37.1-44.1$ & 0.2 \\
\hline Obesidad abdominal & 138 & 59.3 & $50.5-68.1$ & 767 & 37.9 & $34.5-41.3$ & $<0.0001$ \\
\hline
\end{tabular}

${ }^{*} \chi^{2}$. HABLE $=$ Health \& Aging Brain among Latino Elders, ENASEM $=$ Estudio Nacional de Salud y Envejecimiento en México.

Tabla 3. Prevalencia de factores de riesgo cardiovascular por sexo femenino

\begin{tabular}{|c|c|c|c|c|c|c|c|}
\hline & \multicolumn{3}{|c|}{ HABLE } & \multicolumn{3}{|c|}{ ENASEM } & \multirow[t]{2}{*}{$\mathbf{p}^{*}$} \\
\hline & n & $\%$ & IC $95 \%$ & $n$ & $\%$ & IC $95 \%$ & \\
\hline Hipertensión & 421 & 50.6 & $45.9-55.5$ & 7877 & 54.3 & $53.2-55.4$ & 0.1 \\
\hline Diabetes & 421 & 36.8 & $32.2-41.5$ & 7877 & 27.5 & $26.5-28.4$ & $<0.0001$ \\
\hline Hipercolesterolemia & 421 & 45.8 & $41.0-50.6$ & 1083 & 53.3 & $50.3-56.2$ & 0.009 \\
\hline Obesidad abdominal & 421 & 89.6 & $86.6-92.5$ & 1112 & 77.0 & $74.5-79.4$ & $<0.0001$ \\
\hline
\end{tabular}

${ }^{*} \chi^{2} \cdot$ HABLE $=$ Health \& Aging Brain among Latino Elders, ENASEM $=$ Estudio Nacional de Salud y Envejecimiento en México.

de prevalencia de hipertensión arterial entre mujeres de ambos estudios no fue estadísticamente significativa $(p=0.1)$.

\section{Discusión}

Los resultados de nuestro estudio sugieren que los participantes de ENASEM tienen una menor prevalencia de FRCV que los participantes de HABLE. Los hombres de HABLE tuvieron una prevalencia más alta de hipertensión, diabetes y obesidad abdominal que los de ENASEM. Entre las mujeres, el porcentaje con diabetes y obesidad abdominal también fue mayor en HABLE en comparación con las participantes de ENASEM. Los resultados no se modificaron al incluir educación y estado civil en los modelos.

En las mujeres, no todos los factores de riesgo fueron mayores en el estudio de Estados Unidos. El porcentaje de mujeres con nivel de colesterol elevado fue mayor en las mujeres de ENASEM; la explicación podría estar en la dieta o el nivel de actividad física. Esto concuerda con estudios que sugieren que los latinos con bajos niveles de aculturación en Estados Unidos tienen riesgo mayor para hipercolesterolemia mal controlada ${ }^{18}$ incluso se ha sugerido que el único FRCV que mejora con grados altos de aculturación es la dislipidemia. ${ }^{19}$ De cualquier forma se requiere investigación más detallada para dilucidar estos hallazgos.
Los resultados de nuestro estudio concuerdan con los de otros que sugieren que los mexicanos que residen en México tienen riesgo menor de desarrollar FRCV que quienes viven en Estados Unidos y sus descendientes. ${ }^{20,21}$ Si bien los hallazgos de varios análisis han sido contradictorios, en general se ha tratado de relacionarlos con el grado de aculturación. La aculturación se define como el proceso mediante el cual los inmigrantes adoptan la cultura, creencias y prácticas del sitio en el cual residen. ${ }^{22}$ Se ha sugerido que mientras más alto es el grado de aculturación, mayor es el riesgo de desarrollar FRCV. La explicación podría ser que estos sujetos adoptan comportamientos menos saludables ${ }^{23}$ y el alto nivel de estrés psicológico asociado con la inmigración. ${ }^{24}$ Incluso se ha observado que los mexicanos que viven cerca de la frontera con Estados Unidos tienen un mayor riesgo de mortalidad por problemas cardiovasculares cuando se comparan con habitantes de otras regiones de México, ${ }^{25}$ posiblemente por la cercanía e influencia de la cultura al otro lado de la frontera.

Existen varias limitaciones en este estudio: debido al diseño transversal del estudio, no es posible evaluar el efecto que la dieta, actividad física, y el estrés ejercen en la prevalencia de FRCV; para el diagnóstico de diabetes e hipertensión se dependió del reporte de los participantes, lo que podría haber causado subestimación en la prevalencia de estos factores; además, no se controló por otras variables de confusión como el nivel socioeconómico, el acceso a servicios de salud 
o el uso de medicamentos, que podrían tener un efecto importante sobre la prevalencia de FRCV. Algunas de las fortalezas de este estudio son la similitud entre las dos muestras: en ambas se trató de sujetos residentes en la comunidad y seleccionados de forma aleatoria.

\section{Conclusiones}

Nuestro estudio aporta a la escasa literatura sobre la prevalencia de factores de riesgo cardiovascular a ambos lados de la frontera. Nuestro análisis sugiere que la prevalencia de FRCV fue mayor en mexicoamericanos registrados en el estudio HABLE, que en mexicanos del estudio ENASEM. Se necesitan nuevos estudios para investigar los problemas cardiovasculares en mexicanos de ambos países, usando muestras representativas y con características demográficas y socioeconómicas similares. El mejor entendimiento de estos factores de riesgo cardiovascular debe llevar a diseñar intervenciones dirigidas a prevenir y contrarrestar el efecto que estos factores tienen sobre la salud de los mexicanos y mexicoamericanos.

\section{Agradecimientos}

La investigación reportada aquí fue apoyada por el Instituto Nacional del Envejecimiento, parte de los Institutos Nacionales de Salud. El contenido es responsabilidad de los autores y no representa necesariamente la visión oficial de los Institutos Nacionales de Salud. El equipo de investigación quiere agradecer a la comunidad local de Fort Worth y a los participantes de HABLE y ENASEM. Los archivos de datos y la documentación son de uso público y están disponibles en www.ENASEM.org.

\section{Financiamiento}

HABLE es financiado por el Instituto Nacional del Envejecimiento parte de los Institutos Nacionales de Salud de Estados Unidos bajo el premio número R01AG054073. ENASEM es financiado parcialmente por los Institutos Nacionales de Salud/Instituto Nacional del Envejecimiento (NIHR01AG018016) y por el INEGI.

\section{Conflicto de intereses}

Los autores no tienen ningún conflicto de intereses que declarar.

\section{Bibliografía}

1. National Center for Health Statistics. Mortality in the United States, 2017. Data Brief No. 328, Noviembre 2018 [en línea]. EE. UU.: Centers for Disease Control and Prevention; 2018.

2. Office of Minority Health [en línea]. EE. UU.: U.S. Department of Health and Human Services; 2019.

3. Heron M. Deaths: leading causes for 2016. Natl Vital Stat Rep. 2018; 67:1-77.

4. Características de las defunciones registradas en México durante 2017 México: Instituto Nacional de Estadística y Geografía; 2018.

5. Enfermedades cardiovasculares [en línea]. Ginebra: Organización Mundial de la Salud; 2019

6. Las enfermedades cardiovasculares: un problema de salud pública y un reto global. Biomédica. 2011;31.

7. Sánchez-Arias AG, Bobadilla-Serrano ME, Dimas-Altamirano B, Gómez-Ortega M, González-González G. Enfermedad cardiovascular: primera causa de morbilidad en un hospital de tercer nivel. Rev Mex Cardiol. 2016;27:s98-s102.

8. Hunt KJ, Resendez RG, Williams K, Haffner SM, Stern MP, Hazuda H. All-cause and cardiovascular mortality among Mexican American and non-Hispanic white older participants in the San Antonio Heart Study-evidence against the "Hispanic paradox". Am J Epidemiol. 2003;158: 1048-1057.

9. Daviglus ML, Talavera GA, Avilés-Santa ML, Allison M, Cai J, Criqui MH, et al. Prevalence of major cardiovascular risk factors and cardiovascular diseases among Hispanic/Latino individuals of diverse backgrounds in the United States. JAMA. 2012;308:1775-1785.

10. Encuesta Nacional de Salud y Nutrición (ENSANUT) 2012. México: Instituto Nacional de Salud Pública/Secretaría de Salud; 2013.

11. Schagrodsky H, Hernández-Hernández R, Champagne BM, Silva $H$, Vinueza R, Silva Aycaguer LC, et al. CARMELA: assessment of cardiovascular risk in seven Latin American cities. Am J Med. 2008;121:58-65.

12. Orozco-González CN, Cortés-Sanabria L, Viera-Franco JJ, Ramírez-Márquez JJ, Cueto-Manzano AM. Prevalencia de factores de riesgo cardiovascular en trabajadores de la salud. Rev Med Inst Mex Seguro Soc. 2016:54:594-601.

13. Vaeth PAC, Willet DL. Level of acculturation and hypertension among Dallas County Hispanics: findings from the Dallas Heart Study. Ann Epidemiol. 2005:15:373-380.

14. Eamranond PP, Legedza A TR, Diez-Roux AV, Kandula NR, Palmas W, Siscovick DS, et al. Association between language and risk factor levels among Hispanic adults with hypertension, hypercholesterolemia, or diabetes. Am Heart J. 2009:157:53-59.

15. Estudio Nacional de Salud y Envejecimiento en México. México: Instituto Nacional de Estadística y Geografía/Universidad de Pennsylvania/ Universidad de Maryland/Universidad de Wisconsin; 2015.

16. Wong R, Michaels-Obregon A, Palloni A. Cohort Profile: The Mexican Health and Aging Study (MHAS). Int J Epidemiol. 2017;46:1-10.

17. Mex-Cog, Estudio sobre Envejecimiento Cognitivo Vinculado al ENASEM, [2012]. Archivos de Datos y Documentación (uso público). Mex-Cog [Sección A, Sección C, Sección L]. Consultado 2019 mar 20.

18. Eamranond PP, Wee CC, Legedza AT, Marcantanio ER, Leveille SG Acculturation and cardiovascular risk factor control among Hispanic adults in the United States. Public Health Rep. 2009;124:818-824.

19. López L, Peralta CA, Lee A, Zeki-Al Hazzouri A, Hann MN. Impact of acculturation on cardiovascular risk factors among elderly Mexican Americans. Ann Epidemiol. 2014; 24:714-719.

20. Abraído-Lanza AF, Chao MT, Flórez KR. Do healthy behaviors decline with greater acculturation? Implications for the Latino mortality paradox. Soc Sci Med. 2005;61:1243-1255.

21. Lara M, Gamboa C, Kahramanian MI, Morales LS, Bautista DE. Acculturation and Latino health in the United States: a review of the literature and its sociopolitical context. Annu Rev Public Health. 2005;26: 367-397.

22. Thomson MD, Hoffman-Goetz L. Defining and measuring acculturation: A systematic review of public health studies with Hispanic populations in the United States. Soc Sci Med. 2009;69:983-991.

23. Dixon LB, Sundquist J, Winkleby M. Differences in energy, nutrient, and food intakes in a US sample of Mexican American women and men: findings from the Third National Health and Nutrition Examination Survey, 1988-1994. Am J Epidemiol. 2000;152:548-557.

24. Finch B, Vega WA. Acculturation stress, social support, and self-rated health among Latinos in California. J Immigr Health. 2003:5:109-117.

25. Anaya G, Al-Delaimy WK. Effect of the US-Mexico border region in cardiovascular mortality: ecological time trend analysis of Mexican border and non-border municipalities from 1998 to 2012. BMC Public Health. 2017;17:400. 